Die Studie erfasst und beschreibt nahezu alle gesellschaftspolitisch relevanten Kernbereiche der Digitalisierung. So werden neben den digitalen Trends in Politik und Verwaltung auch die Grundlagenthemen wie Infrastruktur, Souveränität, Sicherheit und Datenschutz, aber auch die digitalen Lebenswelten der Bürger (Verwaltung, Arbeit, Verkehr und Mobilität, Umwelt und Energie, Gesundheit und Kultur) in den Fokus der Betrachtung gerückt.

Nach Ansicht der über 500 befragten Experten muss sich das Engagement des Staates unmittelbar auf die Basisthemen „Digitale Infrastruktur", „Digitale Souveränität" und "IT-Sicherheit und Datenschutz" konzentrieren. Im föderalen Kontext sollte der IT-Planungsrat bei der Gestaltung der Digitalisierung eine treibende Rolle als steuernder Moderator, Koordinator und gestaltende Kraft einnehmen. Hier steht die Studie zur Verfügung: $h t t p: / / w w w . c i o . b u n d$. de/SharedDocs/Publikationen/DE/Aktuelles/studie.pdf?_blob=publicationFile

\section{Simulationsstudie „Ersetzendes Scannen“}

Grundsätzlich könnte die digitale Kopie eines Papierbelegs in Zukunft genügen, um in einem Streitfall zu seinem Recht zu kommen. Dies ist das Resultat einer Simulationsstudie, die die Universität Kassel gemeinsam mit der DATEV eG durchgeführt hat und über deren Ergebnisse am 30.102013 informiert wurde. Zwei Tage lang wurden dazu in Nürnberg insgesamt 14 Gerichtsverhandlungen simuliert. In der Mehrzahl der Fälle entschieden die Richter, dass eine elektronische Kopie als Beweis ausreicht, wenn sie richtig eingescannt und je nach Dokumentenklasse eventuell noch digital signiert ist. „Das Relikt Papierablage kann vor dem Hintergrund unserer Studie in Zukunft hoffentlich bald über Bord geworfen werden", zeigt sich Prof. Alexander Roßnagel vom Institut für Wirtschaftsrecht der Universität Kassel zuversichtlich. „Für Millionen von Unternehmen - insbesondere für den Mittelstand - bedeutet das künftig eine enorme Entlastung bei der Einhaltung der gesetzlichen Aufbewahrungsfristen."

Interessant in diesem Zusammenhang könnte insbesondere das Fazit von Ulrich Schwenkert, Vorsitzender Richter am Finanzgericht Berlin-Brandenburg sein, der im Rahmen der Studie die finanzgerichtlichen Streitfälle zu klären hatte: „Im Regelfall dürften selbst die eigenhändig ohne besondere Vorkehrungen eingescannten Belege nicht zu einem Rechtsnachteil führen". In der finanzgerichtlichen Praxis werde sehr häufig mit Belegkopien gearbeitet, ohne dass die Vorlage des Originals gefordert sei. „Dies muss auch für digitale Kopien gelten", meint Schwenkert.

\section{Elektronisch oder Papier ist nicht die Frage}

Für Richter und Rechtsanwälte ist der Umgang mit elektronischen Belegen inzwischen kein Problem mehr. Ob ein Fall gewonnen oder verloren wird, hängt nicht an der Frage, ob das Beweismittel digital oder in Papierform vorliegt. Wichtiger als der Scan selbst ist die Vor- und Nachbereitung, also die Frage, mit welchen Mitteln der Beleg vor Manipulation geschützt wird. Entsprechend erhöht ein sicherer Scan- und Ablageprozess grundsätzlich den Beweiswert. Um für zivilrechtliche Streitfälle, in denen um Verträge, Rechnungen oder Quittungen gestritten wird, gerüstet zu sein, empfiehlt sich ein entsprechendes Verfahren in jedem Fall. Wenn etwa Vertragsoriginale vernichtet werden, sollte die elektronische Kopie zumindest eine automatisch erstellte elektronische Signatur enthalten.
Wichtiger Beitrag für eine juristische Beurteilung Eine umfassende Rechtssicherheit kann die Simulationsstudie zwar nicht schaffen, doch alle Beteiligten erwarten, dass die aus ihr hervorgegangenen Referenzurteile Vorbildwirkung entfalten. „,Da sie in der Fachwelt ernst zu nehmende Einschätzungen dafür sind, ob das jeweils zugrunde liegende Verfahren als rechtssicher zu bewerten ist, bilden die Urteile einen wichtigen Beitrag für eine juristische Beurteilung zum Ersetzenden Scannen", ist Michael Seyd überzeugt, der bei der DATEV als Mitglied der Geschäftsleitung für die strategische Unternehmensentwicklung verantwortlich ist. „Bei einem sorgfältigen Umgang und entsprechender organisatorischer Gestaltung ist es künftig möglich, zumindest unterschriftslose Belege nach einem sicheren Scan und sicherer elektronischer Archivierung zu vernichten, ohne dabei den Beweiswert zu beschneiden."

Simulationsstudien sind ein gängiger Weg, um in Themenfeldern, in denen es noch keine Urteile aus der Praxis gibt, stichhaltige Argumente für die juristische Diskussion zu liefern. In der Nürnberger Studie wurden unter verschiedenen Gesichtspunkten realitätsnahe Streitfälle verhandelt, bei denen sich eine Partei ausschließlich auf elektronische Kopien ursprünglicher Papierbelege berief, die unter Einsatz unterschiedlicher Technik und organisatorischer Vorgaben erzeugt wurden.

\section{ISOC:DE: Balkanisierung des Internet kein geeignetes Konzept für mehr Datenschutz und Datensicherheit}

Anlässlich des „Weltinternettages“ am 29. Oktober 2013 erklärte der Präsident des deutschen Chapters der Internet Society ISOC.DE, Hans Peter Dittler, zu einigen im politischen Raum im Zusammenhang mit der Abhöraffäre der NSA aktuell diskutierten Vorschlägen:

Die Empörung über die Verletzung der Vertraulichkeit der Datenkommunikation und das Abhörens von Telefongesprächen durch fremde Mächte ist nachvollziehbar. Die von Edward Snowden angestoßene Debatte und die nun bekanntgewordenen neuen Tatsachen offenbaren, wie wenig die Bundesrepublik Deutschland und andere Staaten der EU bislang technisch und organisatorisch auf die Verwundbarkeit von Informationstechnik vorbereitet sind und damit die Freiheit der Bürger und die Sicherheit des Staates gefährdet sein kann. Forderungen nach mehr Sicherheit und einem höheren Niveau des Schutzes von Daten in der Telekommunikation und ganz generell im Internet in Deutschland und in ganz Europa sind daher berechtigt. Vorschläge, die diese Ziele jedoch mit untauglichen Mitteln erreichen wollen, sind aber abzulehnen. Besonders, wenn durch falsche Instrumente im Ergebnis lediglich eine Balkanisierung des Internets bewirkt werden kann, würde die Freiheit der Bürger so nicht geschützt, sondern die Offenheit des Internets durch solche Maßnahmen vielmehr gefährdet. Letztlich dienen solch untauglichen Vorschläge eine lediglich symbolischen Politik, die keine nachhaltigen Verbesserungen für die Menschen erreichen kann, aber von ihrer eigenen Konzept- und Ahnungslosigkeit ablenken will. Dieses aber ist abzulehnen.

In diesem Zusammenhang sieht ISOC.DE besonders solche Forderungen als kritisch an, die gesetzliche Regelungen erreichen wollen, die den freien und neutralen Fluss von Datenpaketen im Internet beispielsweise durch eine Pflicht zum "National Routing" begrenzen sollen. Die Idee, dass sich IP-Pakete im Internet - egal ob sie E-Mails, Sprachnachrichten oder andere Inhalte transpor- 